\title{
新皮啡肽类似物的设计、合成、构象 和生物活性的研究*
}

\author{
王 锐 胡晓愚** 嘉 庆姬虹王 勤
}

(兰州大学生物系, 应用有机化学国家重点实验室, 兰州 730000)

\section{关键词 新皮啡肽 固相多肽合成 二维核磁共振谱 红细胞免疫功能 镇痛}

内源性类阿片肽 (Endogenous opioids) 是指由机体产生的具有与吗啡 (鸦片有效成分) 相 似生理作用的一些肽类物质, 包括脑啡肽、内啡肽、强啡肽、皮啡肽和新皮啡肽等, 其作用相当 广泛, 以镇痛方面的研究较多, 但目前对其镇痛的作用原理仍不甚了解. 新皮啡肽 (Deltorphins) ) $^{[1]}$ 是 1989 年从一种南美洲树蛙的皮肤中发现的具有很强镇痛活性的一类神经肽, 它选择性地结 合 $\delta$ 受体. 从文献检索看出, 有关新皮啡肽的整体镇痛活性及对免疫功能的影响等方面的研 究还未见报道, 新皮啡肽 II 的构象的研究也未见报道.

我们根据目前已有的有关新皮啡肽的各种研究结果和有关理论知识, 新设计并用固相多 肽合成法合成了新皮啡肽 I 和 II 的一些类似物. 运用二维核磁共振谱 ( ${ }^{1} \mathrm{H}-{ }^{1} \mathrm{H}$ COSY 和 NOESY) 研究了新皮啡肽 II 及 $\left[\mathrm{L}-\mathrm{Ala}^{2}\right]$ 新皮啡肽 II 在 DMSO 溶液中的构象. 通过对大鼠脊髓蛛网膜 下腔注射后痛阈变化百分数的测定比较了新皮啡肽 $\mathrm{I}$ 及其类似物的镇痛活性. 以外周血 $\mathrm{T}$ 淋 巴细胞的红细胞玫瑰花结形成百分率 (E-RFC) 及红细胞 $\mathrm{C}_{3 b}$ 受体 $\left(R B C-\mathrm{CR}_{1}\right)$ 和免疫复合物花 环率 $(\mathrm{RBC}-\mathrm{IC})$ 为指标, 通过离体和在体实验, 观察新皮啡肽 $\mathrm{I}$ 及其类似物对大鼠免疫功能的影 响. 试图通过以上研究, 为寻找肽类镇痛药物, 了解它们的结构与活性的关系提供理论依据.

\section{1 材料和方法}

\section{1 材料和仪器}

Boc-氨基酸、二环已基碳二亚胺 (DCC), 三氟乙酸等化学试剂购自 SIGMA, Aldrich. 淋 巴细胞分离液 $(d=1.077 \pm 0.002)$ 为上海试剂厂出品, PRMI 1640 完全培养液由本系生物物理 室提供. 氨基酸组分分析仪为日立 835-50 型, 氨基酸序列分析在 $\mathrm{ABI} 440 \mathrm{~A}$ 氨基酸序列分析 仪上测得, HPLC 用 Waters 产品, 质谱在 ZAB-HS 仪上测定. 雄性 Wistar 大鼠( $140 \sim 160 \mathrm{~g})$ 购自甘肃省新医药研究所, $(200 \sim 250 \mathrm{~g})$ 购自北京医科大学科学实验动物部.

\section{2 新皮啡肽 I、 II 及其类似物的合成}

采用 Merrifield 多肽固相合成法合成了下列肽:

1994-10-28 收稿, 1995-02-28 收修改稿

*国家自然科学基金和国家教委优秀年轻教师基金资助项目

**联系人 


\begin{tabular}{|c|c|c|}
\hline ELI, & Tyr-D-Ala-Phe-Asp-Val-Val-Gly- $\mathrm{NH}_{2}$, & 简称 $\mathrm{Asp}^{4}$; \\
\hline$\left[\mathrm{Val}^{4}, \mathrm{Asp}^{5}\right] \mathrm{DELI}$, & Tyr-D-Ala-Phe-Val-Asp-Val-Gly-NH${ }_{2}$, & 简称 $\mathrm{Asp}^{5}$; \\
\hline$\left[\mathrm{Val}^{4}, \operatorname{Asp}^{6}\right] \mathrm{DELI}$, & Tyr-D-Ala-Phe-Val-Val-Asp-Gly-NH${ }_{2}$, & Asp $^{6}$ \\
\hline$\left[\mathrm{Gly}^{4}, \mathrm{Asp}^{7}\right] \mathrm{DELI}$, & Tyr-D-Ala-Phe-Gly-Val-Val-Asp- $\mathrm{NH}_{2}$, & \\
\hline$\left[\mathrm{L}-\mathrm{Ala}{ }^{2}\right] \mathrm{DELI}$ & Tyr-L-Ala-Phe-Asp-Val-Val-Gly-NH ${ }_{2}$, & I; \\
\hline DE & Tyr-D-Ala-Phe-Glu-Val-Val-Gly-NH ${ }_{2}$, & \\
\hline -Ala $\left.{ }^{2}\right]$ DELII, & Tyr-L-Ala-Phe-Glu-Val-Val-Gly-NH & $\operatorname{Ala}^{2} I I$. \\
\hline
\end{tabular}

合成用自制的 BHA 树脂作为载体, 用 DCC 作缩合剂, 三氟乙酸脱 Boc 保护, 肽 - 树脂用 HF 切割后, 冷冻干燥得粗肽, 该粗肽经 Sephadex G10 脱盐后, 再用 Sephadex G25 进行分配 色谱分离得纯肽.

\section{3 新皮啡肽 II 的二维核磁共振谱测定}

DELII 和 $\left[\mathrm{L}-\mathrm{Ala}{ }^{2}\right]$ DELII 的核磁共振谱在 Bruker WM $500 \mathrm{MHz}$ NMR 仪上测得, 用 DMSO$\mathrm{d}_{6}$ 作溶剂, 氨基酸残基和序列的指定用标准二维技术: ${ }^{1} \mathrm{H}-{ }^{1} \mathrm{H} \operatorname{COSY}$ (氢 - 氢相关谱) 和 2DNOESY(二维 NOE 实验); 通过计算机处理得这两个肽的分子模型.

\section{4 新皮啡肽 I 及其类似物的整体镇痛活性的测定}

脊盛蛛网膜下腔埋植瘘管：参照文献[2] 给 $200 \sim 250 \mathrm{~g}$ 雄性 Wistar 大鼠的脊䯣蛛网膜下 腔埋植瘘管。

测痛阈变化：对大鼠给药前后用辐射热甩尾法测痛 ${ }^{[3]}$, 求出痛阈变化百分数.

\section{5 新皮啡肽 I 及其类似物对大鼠免疫功能的影响}

在体实验：肽样品溶液用 $0.01 \mathrm{~mol} / \mathrm{L}, \mathrm{pH} 7.2$ 的 PBS 配制, 取 $140 \sim 160 \mathrm{~g}$ 大鼠 24 只, 随 机分成 8 组, 每组 3 只, 其中 5 组静脉注射 (i.v.) 不同剂量的 DELI, 依次为 $0,0.5,5,50,500 \mu \mathrm{g} / \mathrm{kg}$, 其余 3 组分别 i.v. Asp ${ }^{5}, \mathrm{Asp}^{6}$ 和 $\mathrm{Asp}^{7}$, 剂量均为 $5 \mu \mathrm{g} / \mathrm{kg}, 3 \mathrm{~h}$ 后开始实验.

参照文献[4] 进行外围血 $\mathrm{T}$ 淋巴细胞玫瑰花结试验, 计算活性红细胞玫瑰花结形成细胞百 分率 $\mathrm{E}_{\mathrm{a}}-\mathrm{RFC}$ 和总红细胞玫瑰花结形成细胞百分率 $\mathrm{E}_{\mathrm{t}}-\mathrm{RFC}$. 参照文献[5] 进行红细胞免疫功 能的检测, 计算 $\mathrm{RBC}-\mathrm{CR}_{1}$ 和 RBC-IC.

离体实验: 肽样品溶液用 RPMI 1640 完全培养液配制. 两只大鼠各采血 $1 \mathrm{~mL}$, 分离单 个核细胞, 调整细胞浓度为 $4 \times 10^{6}$ 个 $/ \mathrm{mL}$ 加人, $100 \mu \mathrm{L} /$ 孔 $\times 8$, 其中两孔先加人 $3 \times 10^{-6} \mathrm{~mol} / \mathrm{L}$ 纳洛酮 $50 \mu \mathrm{L}$, 预孵育 $30 \mathrm{~min}$ 后, 分别加人 $0,4 \times 10^{-12} \mathrm{~mol} / \mathrm{L}$ 的 DELI $50 \mu \mathrm{L}$, 其余各孔依次加 人 $0,2 \times 10^{-8}, 2 \times 10^{-10}, 2 \times 10^{-12}, 2 \times 10^{-14}, 2 \times 10^{-16} \mathrm{~mol} / \mathrm{L}$ 的 DELI $100 \mu \mathrm{L}$, 培养 $24 \mathrm{~h}$, 同法 测定 $\mathrm{E}_{\mathrm{a}}-\mathrm{RFC}, \mathrm{E}_{\mathrm{t}}-\mathrm{RFC}$ 和 $\mathrm{RBC}-\mathrm{IC}$.

\section{6 统计学处理}

实验数据均以均值 \pm 标准误差 $(\bar{X} \pm \mathrm{SD})$ 表示, 并用 Student $\mathrm{t}$-test 分析. * 示差异明显, $0.01<P<0.05 ; * *$ 示差异显著, $P<0.01$.

\section{2 结果和讨论}

\section{1 合成肽的鉴定}

所有合成肽经 HPLC 鉴定纯度均大于 $98 \%$, FAB-MS 测其分子量与计算值相符, 氨基酸 组分分析的实测值与理论值相近, 氨基酸序列分析表明合成肽序列与目标肽序列一致. 


\section{2 新皮啡肽 II 在溶液中的构象}

${ }^{1} \mathrm{H}-{ }^{1} \mathrm{H}$ COSY 和 NOESY 分析表明 DELII 和[L-Ala ${ }^{2}$ DELII 在 DMSO-d 6 中有非常相似 的构象, 两者的 C-端四肽均为 $3_{10}$ 螺旋, 即为 III 型转角结构. 根据我们通过计算机建立的这 两个肽的分子模型, 发现两者的唯一差别在于 2 位的丙氨酸的构型不同.

\section{3 新皮啡肽 I 对大鼠免疫功能的影响}

实验表明静脉注射剂量适中的 DELI 能明显促进免疫功能, 高剂量则抑制免疫. 离体条 件实验也表明 DELI 能促进免疫功能, 并有最适浓度范围. 从实验结果发现纳洛酮 $\left(10^{-6} \mathrm{~mol} / \mathrm{L}\right)$ 可以阻断 $10^{-12} \mathrm{~mol} / \mathrm{L}$ 的 DELI 增加 E-RFC 和 $\mathrm{RBC}-\mathrm{CR}_{1}$ 的作用.

\section{4 新皮啡肽 I 及其类似物的结构与活性关系}

免疫活性比较: 实验表明: $A s p^{5}$ 和 $\mathrm{Asp}^{6}$ 与 $\mathrm{Asp}^{4}$ 相比, E-RFC 和 $\mathrm{RBC}-\mathrm{CR}_{1}$ 均明显下 降; 而 $\mathrm{Asp}^{7}$ 与 $\mathrm{Asp}^{4}$ 相比, Ea-RFC 变化不明显, $\mathrm{E}_{\mathrm{t}}-\mathrm{RFC}$ 明显下降, RBC-CR, 却又明显升高; 这几个肽的 RBC-IC 变化不明显. 免疫活性大小为 $\mathrm{Asp}^{4}>\mathrm{Asp}^{7}>\mathrm{Asp}^{6}>\mathrm{Asp}^{5}$.

镇痛活性比较: 将 54 只大鼠随机分成 13 组 (每组 $4 \sim 5$ 只) 在脊髓蛛网膜下腔分别注人 生理盐水、剂量为 $5,10,20 \mathrm{nmol}$ 的 $\mathrm{Asp}^{4}, \mathrm{Asp}^{5}, \mathrm{Asp}^{6}$ 和 $\mathrm{Asp}^{7}$. 结果表明, 三个剂量下 $\mathrm{Asp}^{5}$ 和 $\mathrm{Asp}^{6}$ 的镇痛活性都比 $\mathrm{Asp}^{4}$ 小, $P<0.01$, 差异显著; $\mathrm{Asp}^{7}$ 在 $5 \mathrm{nmol}$ 时活性小于 $\mathrm{Asp}^{4}, P<0.01$, 而在 10 和 $20 \mathrm{nmol}$ 时活性已接近 $\mathrm{Asp}^{4}$. 镇痛活性大小为 $\mathrm{Asp}^{4}>\mathrm{Asp}^{7}>\mathrm{Asp}^{5}>\mathrm{Asp}^{6}$.

\section{5 讨论}

DELI 及其类似物的镇痛活性和免疫活性差别的原因只能从这些肽的结构来分析: Asp 和其后 3 个氨基酸逐次交换位置, 当 Asp 处于 5, 6 位时导致 DELI 的内部氢键等被破坏, 在 空间取向上也就不利于与 $\delta$ 受体结合, 因此镇痛活性和免疫活性都较低. $\mathrm{Asp}^{4}$ 的 C端四肽形 成 1 个 $\beta$-转角结构 ${ }^{[6}$, 而我们设计的 $\mathrm{Asp}^{7}$ 的 C 端四肽: $\mathrm{Gly}^{4}-\mathrm{Val}^{5}-\mathrm{Val}^{6}-\mathrm{Asp}^{7}-\mathrm{NH}_{2}$ 正好与 $\mathrm{Asp}^{4}$ 的 $\mathrm{C}$ 端四肽的序列相反, 可能也具有 $\beta$-转角结构, 都能与 $\delta$ 受体结合, 所以镇痛活性和免疫活性 都较高. DELII 的 C 端四肽具有 III 型转角结构, 故其能与 $\delta$ 受体很好地结合就不难理解了, 并可以预见其具有强的镇痛活性和免疫活性.

致谢 本工作镇痛实验部分得到北京医科大学韩济生院士的指导帮助, 分子模型计算 机处理部分得到北京大学化学系来鲁华教授的帮助,特此一并致谢.

\section{参 考 文 軒}

1 Erspamer V, Melchiorri P, Falconieri-Erspamer G et al. Deltorphins: a family of naturally occurring peptides with high affinity and selectivity for $\delta$ opioid binding sites. Proc Natl Acad Sci USA, 1989, 86:5188 5192

2 Brodeur G M, Seeger R C, Schwab M et al. Amplification of $\mathrm{N}$-myc in untreated human neuroblastomas correlates with advanced disease stage. Science, 1984, 224:1 121 1 124

3 朴素芬, 韩济生. 大鼠杏仁核内注射八肽胆妻收缩素 (ock-8) 拮抗吗啡镇痛的研究. 生理学报, 1993, 45(5): 470 478

4 上海市医学化验所. 临床免疫学检验 (上). 上海：上海科技出版社, 1983

5 郭 峰, 處紫茜, 赵中平. 红细胞免疫功能的初步研究. 中华医学杂志, 1982, 62:715

6 Amodeo P, Motta A, Tancredi $\mathrm{T}$ et al. Solution structure of deltorphin I at 265K: a quantitative NMR study. Peptide Res, 1992, 5: 48 